\title{
Stephen J. Shoemaker, The Death of a Prophet. The End of Muhammad's Life and the Beginning of Islam
}

Philadelphia, University of Pennsylvania Press, 2012, 408 p.

\section{Hassan Bouali}

\section{(2) OpenEdition \\ Journals}

\section{Édition électronique}

URL : http://journals.openedition.org/assr/26558

DOI : $10.4000 /$ assr.26558

ISSN : $1777-5825$

\section{Éditeur}

Éditions de l'EHESS

Édition imprimée

Date de publication : 31 décembre 2014

Pagination : 288

ISBN : 978-2-7132-2467-6

ISSN : 0335-5985

\section{Référence électronique}

Hassan Bouali, «Stephen J. Shoemaker, The Death of a Prophet. The End of Muhammad's Life and the Beginning of Islam », Archives de sciences sociales des religions [En ligne], 168 | 2014, mis en ligne le 21 mai 2015, consulté le 22 septembre 2020. URL : http://journals.openedition.org/assr/26558 ; DOI : https://doi.org/10.4000/assr.26558 


\section{Stephen J. Shoemaker, The Death of a Prophet. The End of Muhammad's Life and the Beginning of Islam}

Philadelphia, University of Pennsylvania Press, 2012, 408 p.

Hassan Bouali

\section{RÉFÉRENCE}

Stephen J. Shoemaker, The Death of a Prophet. The End of Muhammad's Life and the Beginning of Islam, Philadelphia, University of Pennsylvania Press, 2012, 408 p. 
1 Avec cet ouvrage, Stephen J. Shoemaker (SJS), qui est avant tout un spécialiste du Christianisme, en particulier des traditions primitives concernant la Vierge Marie (The Ancient Traditions of the Virgin Mary's Domition and Assumption, Oxford University Press, 2002 ; The Life of the Virgin attributed to Maximus the Confessor, Yale University Press, 2012), se lance un véritable défi, en réorientant ses recherches vers l'histoire et l'historiographie des débuts de l'islam. L'auteur se propose de discuter la date traditionnellement retenue pour la mort de Muhammad (632). Pour ce faire, il inscrit son investigation dans le sillage du Hagarism de M. Cook et P. Crone (Hagarism The Making of The Islamic World, Cambridge University Press, 1977), lesquels ont

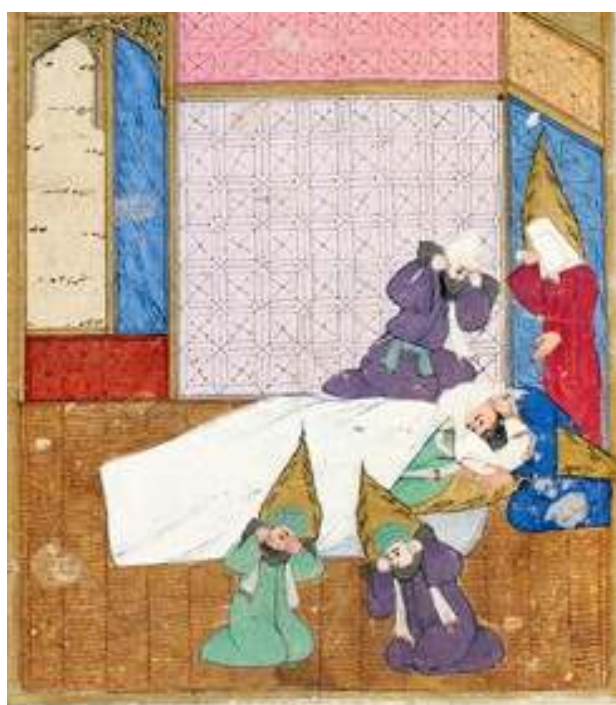

The Death of a Prophet

This End of A lutarmants tife and the Reginninger of blem Stephen I. Shoemaker identifié un certain nombre de sources non musulmanes $\mathrm{du} \mathrm{VII}^{\mathrm{e}}-\mathrm{VIII}{ }^{\mathrm{e}}$ siècle qui indiquent que Muhammad était encore vivant en 634, et qu'il a lui-même mené l'expédition initiale des conquêtes islamiques.

2 La première partie de l'ouvrage («A Prophet Has appeared, Coming with the Saracens: Muhammad's Leadership during the Conquest of Palestine According to Seventh - and Eight Century Sources ", p. 18-72) analyse les différentes sources qui remettent en cause la date traditionnellement retenue pour la mort de Muhammad. Tour à tour, la Doctrina Jacobi, l'Apocalypse du rabin Shi'môn b. Yohai, la chronique Khûzistâne (660), celle de Jacob d'Edesse (691/692), l'Histoire des patriarches d'Alexandrie (avant 717), la " source orientale espagnole » (741), la « source commune syriaque » (un texte préservé dans la chronique de 1234 dont Théophile d'Edesse est vraisemblablement l'auteur), la chronique syriaque de 775 (auteur anonyme), la chronique de Zuqnin (775; auteur anonyme), la chronique samaritaine (compilée au plus tard en 1355 à partir d'un matériau de sources anciennes), sont analysées par l'auteur. Il en ressort que ces textes affirment, chacun à leur façon, que le Prophète Muhammad était encore vivant en 634, et qu'il dirigea les premières incursions arabes hors d'Arabie. Par exemple, l'auteur de la Doctrina Jacobi évoque le "Prophète qui est apparu avec les Saracènes » (p. 22 ; voir aussi A. L. De Prémare, Les fondations de l'islam. Entre écriture et histoire, Le Seuil, 2002, p. 131) tandis que celui de la chronique Khûzistâne, note que Muhammad dirigea lui-même les incursions contre les Perses (p.34; ibid., p.132). Qu'en est-il de la onzième source retenue par S. J. Shoemaker? Du fait de son origine islamique, celle-ci est sans aucun doute la plus originale. Il s'agit d'une lettre attribuée au calife omeyyade Umar 2 (717-720) et adressée à l'empereur byzantin Léon III (717-741). Dans cette lettre de défense de l'islam vraisemblablement écrite avant la fin $\mathrm{du}$ vIII ${ }^{\mathrm{e}}$ siècle, dans un contexte marqué par l'émergence d'un genre littéraire qui met en scène des correspondances polémiques entre Umar 2 et Léon III, le Prophète Muhammad est présenté comme celui qui est sorti d'Arabie pour affronter les Byzantins et les Perses. Selon l'auteur, qui 
s'appuie sur les travaux de R. Hoyland (R. Hoyland, Seeing Islam as Others saw it. A Survey and Evaluation of Christian, Jewish and Zoroastrian Writings on Early Islam, Darwin Press, 1997) et Gaudeul (Gaudeul, «The Correspondance between Léo and Umar: Umar's Letter Re-discovered?», Islamochristiana 10, 1984, p. 109-157), pour identifier l'origine syrienne de la chaîne de transmission, cette lettre est un document précieux qui confirme ce que disent les sources non musulmanes, au moment même où une biographie officielle du Prophète émerge sous le patronage des Abbassides.

3 Dans le second chapitre, l'auteur mène une analyse "hypercritique " des sources musulmanes qui exposent la vie du Prophète ( $«$ The End of Muhammad's Life in Early Islamic Memory: The Witness of the Sîra Tradition », p. 73-117). L'auteur présente tout d'abord les sources les plus anciennes. Parmi elles, la biographie rédigée par Ibn Ishâq (m. 767) occupe une place de choix. Shoemaker pointe du doigt le fait que cette source, aujourd'hui perdue mais connue grâce à des recensions tardives par Ibn Hichâm (m. 833) et al-Tabarî (m. 923), a été compilée plus de 120 ans après la mort de Muhammad. Que dire des biographies de Mûsâ b. Uqba (m. 758) et Ma'mar b. râshid (m. 770; voir l'édition et la traduction récente de S. W. Anthony, Ma'mar b. râchid.The Expeditions. An Early Biography of Muhammad, New York University Press, 2014), tous deux disciples du traditionniste médinois al-Zuhrî (m. 742) ? Leur matériau, lui aussi perdu, a survécu par fragments dans des œuvres postérieures. Le traditionniste médinois al-Wâqidi (m. 822) est aussi censé avoir recueilli un ensemble de traditions relatives à la mort du Prophète (Kitâb wafât al-nabî), mais il ne subsiste que dans les œuvres de son élève Ibn Sa'd (m. 845). L'auteur stigmatise ensuite des œuvres telles que le Musannaf d'Ibn Abî Shayba (m. 849) et le Sahîh d'al-Bukhârî (m. 870). Selon lui, ces compilations de hadiths, bien que rapportant des récits ayant trait à la mort du Prophète, ne s'appuient pas sur des traditions attestées par la première littérature islamique. S. J. Shoemaker dresse un bilan bien plus mitigé encore des biographies postérieures à ces premières sources. Tout en reconnaissant la possibilité que ces collections tardives contiennent des traditions antérieures, il met en avant la fréquence des forgeries et des fausses attributions à des auteurs réputés (tels qu'al-Zuhrî et Urwa b. al-Zubayr). Le récit d'Ibn Ishâq et ceux attribués à al-Zuhrî, sur la mort et l'enterrement de Muhammad, sont également disséqués. Il en ressort que le premier s'est vraisemblablement appuyé sur des traditions rapportées par le second. L'auteur stigmatise enfin la chronologie de ces récits biographiques, laquelle, en plus d'être artificielle, n'a pas existé avant le milieu du viII ${ }^{\mathrm{e}}$ siècle.

Le troisième chapitre se focalise sur la nature du mouvement religieux fondé par Muhammad. L'auteur présente d'emblée ce dernier comme un Prophète eschatologique qui croyait en l'imminence de la fin des temps («The Beginnings of Islam and the End of Days. Muhammad as Eschatological Prophet», p.118-196). Après un bilan historiographique de cette question («The Eschatological Prophet of Early Western Scholarship ", p. 121-127; "Bell, Watt, and the Non Eschatological Prophet of Social Reform ", p. 127-136), l'historien américain étaye son point de vue en s'appuiyant sur l'eschatologie coranique puis sur celle véhiculée par la Tradition «primitive" («Imminent Eschatology in the Early Islamic Tradition», p.172-178). Pour lui, le mouvement religieux de Muhammad a connu une «réorientation radicale " (p. 195) suite aux espoirs déçus d'une arrivée imminente de la fin des temps avant la mort de celui-ci. Ce qui ne fut pas sans entraîner une révision historique de ses origines, 
notamment en relocalisant la mort du Prophète dans la Péninsule arabique, bien que celle-ci ait eu lieu ailleurs.

Dans le dernier chapitre, l'auteur établit un lien étroit entre la mutation du mouvement religieux fondé par Muhammad et la création d'une nouvelle géographie sacrée dans l'islam premier ( From Believers to Muslims, Jerusalem to The Hijâz: Confessionnal Identity and Sacred Geography in Early Islam»). L'auteur entend montrer que le mouvement religieux fondé par le Prophète était interconfessionnel. Pour ce faire, il s'appuie sur des sources non musulmanes telles que la chronique arménienne (vers 660) attribuée à l'évêque Sebeos et Les secrets du rabin Shim'ôn b. Yohai. L'exemple de cette dernière est symptomatique. Écrite dans un milieu juif, cette œuvre présente l'avènement du « royaume d'Ismaël » (p. 203) en Terre sainte comme une volonté de la providence divine. Pour conforter cette idée, S. J. Shoemaker s'appuie aussi sur la fameuse Constitution de Médine (voir M. Lecker, The "Constitution of Medina" Muhammad's First Legal Document, Darwin Press, 2004), dans le sillage des travaux de F. Donner (Donner, « From Believers to Muslims: Confessional Self-Identity in The Early Islamic-Community ", Al-Abhath 50-51, 2002-2003, p. 9-53; Donner, Muhammad and The Believers: At the Origins of Islam, The Belknap Press of Harvard University Press, 2010). Selon l'auteur, la libération de la Terre sainte était au cœur de l'enseignement du Prophète, d'où le statut privilégié de Jérusalem, dans la géographie sacrée de l'islam premier. En témoigne, la manière dont le Coran exalte cette Terre sainte (al-ard almuqadasata), mais aussi le fait que Muhammad et ses partisans prièrent tout d'abord vers Jérusalem. Suivant cette lecture, l'auteur émet l'idée que Muhammad a probablement appelé ses partisans à conquérir la Terre que Dieu a promise (voir De Prémare, Les fondations de l'islam, op. cit., chap. 7 « Terre promise », p. 131-151) aux « fils d'Abraham» (p. 218). Le mouvement interconfessionnel fondé par Muhammad se distingua néanmoins des autres religions monothéistes du Proche-Orient, sous l'impulsion du calife omeyyade Abd al-Malik (692-705; pour ce personnage, voir Chase F. Robinson, 'Abd al-Malik, Oneworld Press, 2007 ; A. Borrut, Entre mémoire et pouvoir : l'espace syrien sous les derniers Omeyyades et les premiers Abasssides, Brill, 2011), non sans conséquence. En effet, à l'heure où le souverain omeyyade organisait l'islamisation de l'État arabe et l'arabisation de l'islam, il était nécessaire d'imposer une Terre sacrée arabe. Cette politique, qui fut d'ailleurs poursuivie, de manière décisive, par la dynastie abbasside, consacra entièrement le Hijâz comme un espace sacré de l'islam. Il fallait donc nécessairement inscrire la mort du Prophète à Médine, même si elle avait eu lieu ailleurs, pour conforter cette entreprise de sacralisation du Hijâz. C'est ainsi qu'émergèrent progressivement des lieux de pèlerinage dans la Péninsule arabique (la tombe du Prophète par exemple). Pour conforter sa thèse, Shoemaker ne manque pas de stigmatiser l'origine médinoise des auteurs qui ont rédigé la biographie du Prophète. Selon lui, ces auteurs ont indéniablement voulu faire du Hijâz, leur région d'origine, le lieu privilégié de la geste muhammadienne.

6 L'auteur conclut en affirmant l'intérêt qu'il y a d'utiliser l'approche méthodologique des études bibliques, pour appréhender les premières biographies islamiques de Muhammad. Pour lui, celles-ci ressemblent en tout point aux Actes apocryphes des apôtres.

7 La thèse de l'auteur inscrit indéniablement celui-ci dans le camp des historiens dits "sceptiques ", dans la continuité des travaux de M. Cook et P. Crone (Hagarism, op. cit.; voir aussi Crone, Meccan Trade and the Rise of Islam, Princeton University Press, 1987), 
J. Wansbrough (The Sectarian Milieu, Oxford University Press, 1978) ou encore J. Koren et Y. Nevo (Crossroads to islam. The Origins of the Arab Religion and the Arab State, Prometheus Book, 2003). La démarche n'en demeure pas moins iconoclaste. Si les problèmes spécifiques des sources islamiques ont été mis en évidence, de très longue date, par les « orientalistes » (tels que L. Caetani, Annali dell'Islam, U. Hoepli, 1905-1926 ; I. Goldhizer, Muhammedanische Studien, Max Niemayer, 1889-90 ; H. Lammens, Fatima et les filles de Mahomet: notes critiques pour l'étude de la Sîra, Sumptibus Pontificii Instituti Biblici, 1912), ces derniers ne rejettent pas pour autant, dans leur grande majorité (voir F. E. Peters, Muhammad and the Origins of Islam, State University of New York, 1994; M. Watt, Muhammad at Medina, 1956), la date traditionnellement retenue pour la mort du Prophète (632). Même F. Donner, qui a largement influencé Shoemaker dans ses réflexions sur le "mouvement des Croyants ", ne remet pas en question cette datation traditionnelle («Following the death of Muhammad in 11/632, the Believers quickly [...] resolved the question of leadership of the community... », Donner, Muhammad and the Believers, op. cit., p. 90). Que dire d'A.L. De Prémare, qui se garde de trancher cette épineuse question, en préférant parler d'un "problème chronologique en suspens » (De Prémare, Les fondations de l'islam, op. cit., p. 132)?

8 De fait, la thèse de l'auteur, aussi audacieuse soit-elle, pose problème pour plusieurs raisons, dont celles-ci :

9 L'auteur clame dès l'introduction, la dette qu'il doit au travail colossal (p. 3-4) que R. Hoyland a consacré aux sources non musulmanes qui traitent des débuts de l'islam. Malheureusement, bien qu'il déclare inscrire son approche méthodologique des sources non islamiques (identifier la source, le caractère et le sujet de l'observation, voir Hoyland, "Using Non-Muslim Sources: An argumentative Approach ", in Seeing Islam as Others saw it, op. cit., p. 591-598) dans les pas de R. Hoyland, pour établir leur valeur historique, S. J. Shoemaker ne respecte pas toujours ce vœu pieux, loin s'en faut même, comme il le reconnaitt d'ailleurs en certains endroits (p. 35). Il ressort de cet écueil que l'auteur omet de prendre rigoureusement en considération, le caractère apologétique des phénomènes décrits par les sources non musulmanes qui parlent d'une possible présence du Prophète en Palestine, en 634. Par exemple, ne faut-il pas penser, à la suite de J. Tolan, que l'évocation, par la Doctrina Jacobi, du « Prophète qui est apparu avec les Saracènes » (p. 22), est une façon de présenter les conquérants arabes comme «les acteurs du drame divin des derniers jours" (Tolan, Les sarrasins. L'islam dans l'imagination européenne au Moyen Âge, 2003, p. 84)? On comprend donc que l'auteur accorde un crédit sans faille à des sources qui, bien que contemporaines ou presque des premières conquêtes arabes, doivent être, comme le dit R. Hoyland, traitées avec circonspection (R. Hoyland, Seeing Islam as Others saw it, op. cit., p. 595).

Pour étayer sa thèse principale, Shoemaker discrédite trop rapidement les travaux de chercheurs qui ont consacré des années d'études à la biographie du Prophète Muhammad. Ainsi en est-il par exemple avec les travaux de G. Schoeler, H. Motzki et A. Görke (voir A. Görke, H. Motzki, G. Schoeler, «First Century Sources for The Life of Muhammad? A Debate", Der Islam 89 (2), 2012, p. 2-59; A. Görke, G. Schoeler, "Reconstructing the Sîra Texts: The Hijra in the Corpus of Urwa b. al-Zubayr ", Der Islam 82, 2005, p. 209-220; G. Schoeler, The Biography of Muhammad. Nature and Authenticity, Routledge, 2011), lesquels ont montré qu'un certain nombre de traditions peuvent être attribuées à Urwa b. al-Zubayr (m. en 711 ou 712/13), cousin du Prophète et neveu de son épouse Aïcha. 
11 SJS ne tient pas assez compte du fait que le Hijâz, en particulier Médine, a été pour partie sacralisé du vivant même de Muhammad (voir H. Munt, The Holy City of Medina. Sacred Space in Early Islamic Arabia, Cambridge University Press, 2014).

12 La thèse de Shoemaker n'est donc pas convaincante. Certes, l'auteur a raison de pointer $\mathrm{du}$ doigt les limites des sources narratives islamiques. Il a toutefois tort d'accorder une confiance sans faille à des sources qui peuvent être, elles aussi, l'objet du même discrédit. L'ouvrage de SJS a toutefois un mérite: celui de stimuler le débat historiographique relatif aux débuts de l'islam. 